\title{
Lepton Flavor Violation at muon-electron colliders
}

\section{Fabio Bossi ${ }^{a}$ and Paolo Ciafaloni ${ }^{b}$}

${ }^{a}$ INFN - Laboratori Nazionali di Frascati, Via Enrico Fermi 54, I-00044, Italy

${ }^{b}$ INFN - Sezione di Lecce, Via per Arnesano, I-73100 Lecce, Italy

E-mail: fabio.bossi@lnf.infn.it, paolo.ciafaloni@le.infn.it

ABSTRACT: Lepton Flavor Violating (LFV) processes are clear signals of physics beyond the Standard Model. We investigate the possibility of measuring this kind of processes at present and foreseeable future muon-electron colliders, taking into account present day bounds from existing experiments. As a model of new physics we consider a Z' boson with a $U^{\prime}(1)$ gauge symmetry and generic couplings. Processes that violate lepton flavor by two units seem to be particularly promising.

KeYwords: Beyond Standard Model, Global Symmetries

ARXIV EPRINT: 2003.03997 


\section{Contents}

1 Electron-muon collisions $\quad 1$

2 Physics case $\quad 2$

3 Low energy bounds on Z' couplings 3

$4 \mathrm{e}^{+} \boldsymbol{\mu}^{-} \rightarrow \mathrm{e}^{-} \boldsymbol{\mu}^{+}$and $\mathrm{e}^{-} \boldsymbol{\mu}^{+} \rightarrow \mathrm{e}^{+} \boldsymbol{\mu}^{+}$

$\begin{array}{llr}5 & \text { Other processes } & 7\end{array}$

$\begin{array}{llr}6 & \text { Conclusions } & 8\end{array}$

\section{Electron-muon collisions}

Electron-muon collisions have been so far studied using the interaction of a muon beam with a fixed target. Muon beams can have energies up to several hundreds GeV, fluxes of $10^{7}-10^{8}$ particles per second and transverse dimensions of order of a few centimeters [1-3]. Although with this technique one can in principle reach very high luminosities by the use of properly studied targets, the available center of mass energy (c.m.e.) is strongly suppressed because of the Lorenz boost.

In recent years, however, a very intense $R \& D$ program has been put forward in many laboratories to develop techniques to obtain very high energy muon-muon interactions in collider mode, motivated by the possibility of producing collisions of point-like particles at very high energy without the limitation from synchrotron radiation typical of electronpositron machines. In these studies muons are produced either as decay products of pions from fixed-target proton-proton collisions, or as a result of pair production in electronpositron collisions. Typically, at c.m.e. of $6 \mathrm{TeV}$, two single bunches of $2 \times 10^{12}$ muons, each of transverse dimensions of $\sim 1.5 \mu \mathrm{m}$, collide at a rate of $\sim 50 \mathrm{kHz}$, providing a peak luminosity largely exceeding $10^{34} \mathrm{~cm}^{-2} \mathrm{~s}^{-1}[4-7]$. One of the main technical limitations to obtain high luminosity comes from the difficulty in producing highly collimated muon beams. A big step forward in this direction has been recently reported by the MICE collaboration which has been able to confirm the success of a ionization cooling experiment on a low momentum muon beam using properly designed absorbers [8]. This experiment has to be considered as an important advance in the development of high brightness muon beams.

In this paper we want to call the attention to the fact that such beams could also be used to produce electron-muon collisions in collider mode, by the simultaneous usage of high energy electron/positron beams. This would allow probing electron-muon interactions at c.m.e orders of magnitude higher with respect to the fixed target option. 
Actually, electron and positron beams with energies up to $100 \mathrm{GeV}$ have been already put in collision at LEP [9]. Projects for the construction of future linear or circular $\mathrm{e}^{+} \mathrm{e}^{-}$ colliders with c.m.e. between 200 and $1500 \mathrm{GeV}$ and luminosities exceeding $10^{34} \mathrm{~cm}^{-2} \mathrm{~s}^{-1}$ have been also put forward, limited mostly by budget issues. These projects vary a lot among each other in the strategy to reach high luminosity, which is obtained either by maximising the rate of collisions $\left(f_{c}\right)$ or the number of particles in each bunch $\left(N_{l}\right)$ or by minimising the beams dimensions $\left(\sigma_{x, y}\right)$; typical figures of merit are $f_{c} \geq 100 \mathrm{kHz}$, $N_{l} \sim 10^{11}-10^{12}, \sigma_{x, y} \leq 1 \mu \mathrm{m}[10-12]$.

For two colliding beams of particles of type A and B the luminosity can be computed to good approximation by the formula:

$$
\frac{N_{A} \cdot N_{B}}{2 \pi \sqrt{\sigma_{A x}^{2}+\sigma_{B x}^{2}} \sqrt{\sigma_{A y}^{2}+\sigma_{B y}^{2}}} \times f_{c}
$$

Using the numbers quoted above we see that, by properly combining the aforementioned lepton beams, it is at least in principle conceavable to obtain electron(positron)muon collisions at a luminosity of $10^{34} \mathrm{~cm}^{-2} \mathrm{~s}^{-1}$ or more, in a c.m.e. range between few hundreds to few thousands GeV.

A thorough discussion of the technical details of this hypothetical machine goes well beyond the purpose of our paper. We note, however, that since the collision rate would likely be limited by the revolution rate of the muon beam, reaching high luminosity must rely on the ability of producing very compact and dense lepton bunches. We note also that the scheme in [7] naturally provides a source for both muon and positron beams, although the latter must be accelerated at higher energies in a subsequent stage.

\section{Physics case}

Although in principle many interesting measurements can be performed with the above mentioned machine, we focus our attention on the possibility of studying $\mathrm{e}^{+(-)} \mu^{-(+)} \rightarrow$ $\mathrm{e}^{-(+)} \mu^{+(-)}$transitions. The observation of this process would be a clear signature of Lepton-Flavor-Violation (LFV). In the Standard Model (SM) LFV processes are suppressed to an unobservable level, therefore the observation of the aforementioned events would be a clear signature of physics Beyond the Standard Model (BSM).

Many specific and well motivated BSM models including LFV can be found in literature (see for instance [13-15]; see also [16, 17] and references therein). We are however not interested in discussing them in detail, but will rather use a simple model where LFV transitions are mediated by a generic heavy neutral boson (Z') with mass and coupling to be determined by the experiment. Our purpose is, in fact, to give an order-of-magnitude estimate of the potentials and the limits of the proposed experiment as a function of the reachable c.m.e. and luminosity.

We introduce a new LFV interaction mediated by a neutral Z' boson of mass $\mathrm{M}_{z^{\prime}} \gg$ $\mathrm{M}_{z}$, described by the following lagrangian:

$$
g_{i j}^{e L} \bar{e}_{i} Z^{\prime} P_{L} e_{j}+g_{i j}^{e R} \bar{e}_{i} Z^{\prime} P_{R} e_{j}+g_{i j}^{\nu L} \overline{\nu_{i}} Z^{\prime} P_{L} \nu_{j}+g_{i j}^{\nu R} \overline{\nu_{i}} Z^{\prime} P_{R} \nu_{j}
$$




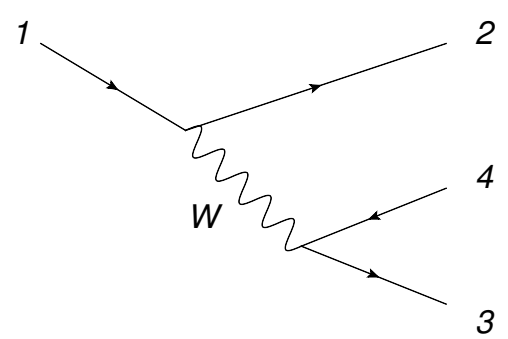

(a)

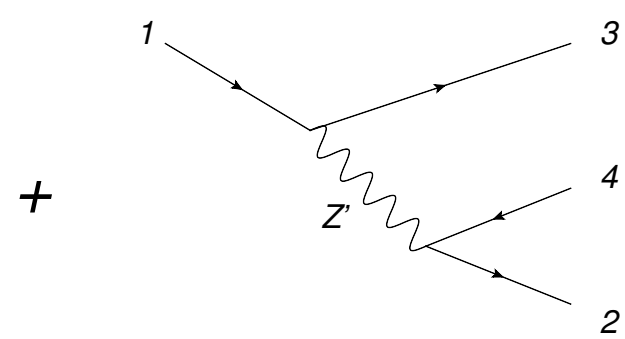

(b)

Figure 1. W and Z' exchange diagrams for the amplitude of a generic decay $1 \rightarrow 2+3+4$. In the case of 'standard' muon decay we have $1=\mu^{-}, 2=\nu_{\mu}, 3=e^{-}, 4=\bar{\nu}_{\mu}$. In the case of LFV muon decay, $1=\mu^{-}, 2=e^{-}, 3=e^{-}, 4=e^{+}$; in this case only the $\mathrm{Z}$ ' exchange diagram contributes.

We take the Z' to be a gauge singlet; then $\mathrm{SU}(2)_{L}$ invariance implies that $\mathrm{g}_{i j}^{e L}=\mathrm{g}_{i j}^{\nu L}$. We do not make any assumption on the couplings of the $Z$ ' with quarks, therefore our results are not affected by the recent $\mathrm{LHCb}$ indications for the possible violation of lepton universality [18]. We only note that this result can be explained through a LFV Z', as discussed in [19].

\section{Low energy bounds on Z' couplings}

Z' exchange amplitudes generate LFV violating processes like $\mu^{-} \rightarrow e^{-} e^{-} e^{+}$, but also contribute to 'standard' non LFV-violating processes like muon decay $\mu^{-} \rightarrow e^{-} \bar{\nu}_{e} \nu_{\mu}$ (see figure 1). In the latter case, the decay width is given by the sum of three terms corresponding to $\mathrm{W}$ exchange square, Z' exchange square and interference; we obtain:

$$
\frac{\Gamma_{\mu}}{m_{\mu}^{5}}=\frac{G_{F}^{2}}{192 \pi^{3}}-\frac{4 \sqrt{2}}{1536 \pi^{3}} \frac{G_{F}\left(g_{\mu e}^{L}\right)^{2}}{M_{Z^{\prime}}^{2}}+\frac{\left[\left(g_{\mu e}^{L}\right)^{2}+\left(g_{\mu e}^{R}\right)^{2}\right]\left[\left(g_{\mu e}^{L}\right)^{2}+\left(g_{\nu_{\mu} \nu_{e}}^{R}\right)^{2}\right]}{1536 \pi^{3} M_{Z^{\prime}}^{4}}
$$

Corrections to the Fermi constant, defined through this process, must be below the per-mille level in order to avoid conflicts with electroweak precision data. We conservatively demand the impact on the Fermi constant to be one order of magnitude smaller, ${ }^{1}$ namely:

$$
\left|B R\left(\mu^{-} \rightarrow e^{-} \bar{\nu}_{e} \nu_{\mu}\right)-B R\left(\mu^{-} \rightarrow e^{-} \bar{\nu}_{e} \nu_{\mu}\right)_{S M}\right| \leq \times 10^{-4}
$$

Notice that one can basically evade the bounds coming from this request by making suitable assumptions on the chirality of the couplings. Indeed, if the Z' mass is of the order of $1 \mathrm{TeV}$ as we assume in the present paper, the third term in (3.1) is suppressed by $\frac{M_{W}^{2}}{M_{Z^{\prime}}^{2}} \approx 10^{-2}$ with respect to the second one. If the $\mu$-e-Z' coupling is purely right $\left(g_{\mu e}^{L}=0\right)$, then the second term is 0 and we obtain very weak bounds on $g_{\mu e}^{R}$. Howewer, in order to be conservative, we assume instead all couplings to be the same $g_{i j}^{L, R}=g \forall i, j$ and obtain stronger bounds. The resulting upper bound on $g$ is plotted on the dashed red line in figure 2. Moreover, we

\footnotetext{
${ }^{1} \mathrm{~A}$ more refined analysis would imply a precise evaluation of the impact of the Z' contribution on the electroweak precision tests; this goes beyond the scope of the present paper.
} 


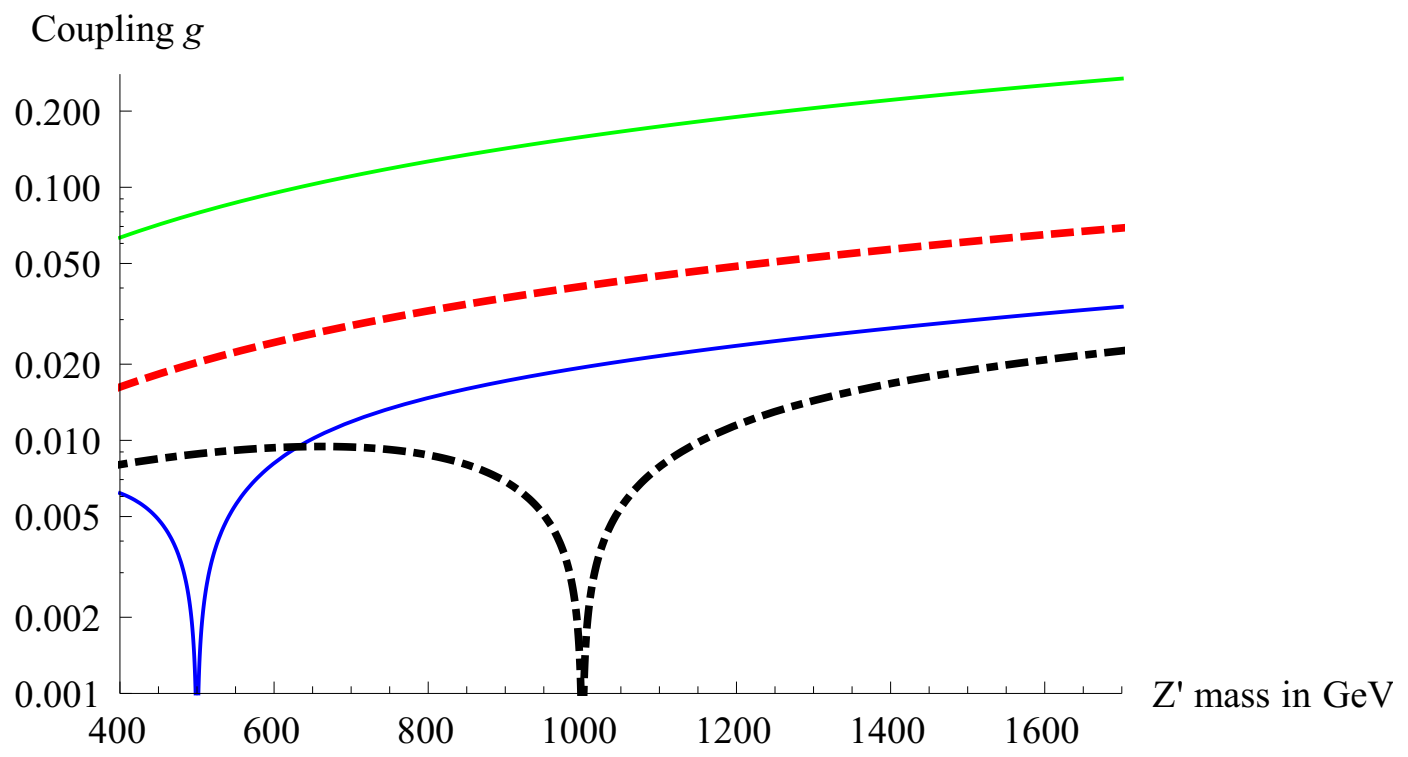

Figure 2. Upper bound on $g_{\mu e}$ as a function of $M_{Z^{\prime}}$ coming from muon decay (red dashed curve) and from muonium-antimuonium oscillation (green continuous line). Value of $g_{\mu e}$ that corresponds to one event/year as a function of $M_{Z^{\prime}}$ with luminosity $\mathcal{L}=10^{34} \mathrm{~cm}^{-2} \mathrm{~s}^{-1}$ and c.m.e. of $500 \mathrm{GeV}$ (blue continuous line) and $1 \mathrm{TeV}$ (dot-dashed line). Left and right couplings are assumed to be equal.

have considered the bounds on $\mathrm{g}$ coming from muonium-antimuon oscillation. Using the effective Lagrangian generated by eq. (2.1) we obtain the oscillation probability [20-22]:

$$
\left.P(\bar{M} u-M u) \approx 10^{-9}\left(\frac{1 T e V}{M_{Z^{\prime}}}\right)^{4} \mid 4\left(g_{\mu e}^{L}\right)^{2}+4\left(g_{\mu e}^{R}\right)^{2}\right)\left.\right|^{2}
$$

Comparing with the present experimental bound [23]

$$
P(\bar{M} u-M u) \leq 8.2 \times 10^{-11}
$$

we obtain:

$$
\left.\mid\left(g_{\mu e}^{L}\right)^{2}+\left(g_{\mu e}^{R}\right)^{2}\right) \mid \lesssim 0.07\left(\frac{M_{Z^{\prime}}}{1 T e V}\right)^{2}
$$

This bound is drawn in figure 2 together with the bound coming from muon decay assuming that left and right couplings are equal. Notice however that the abovementioned bounds depend on different combinations of the couplings (compare (3.1) with (3.3)): for instance, if $g_{\mu e}^{L}=0$ the bound from muon decay is evaded but muonium-antimuniom oscillation still puts bound on the value of $g_{\mu e}^{R}$.

Let us now discuss the bounds coming from the LFV violating process $\mu^{-} \rightarrow e^{-} e^{+} e^{-}$, for which the current experimental bound [24] is $B R<10^{-12}$. In this case only the Z' exchange contributes and assuming $g_{\mu e}^{L}=g_{\mu e}^{R}=g_{\mu e}, g_{e e}^{L}=g_{e e}^{R}=g_{e e}$ we obtain:

$$
\Gamma\left(\mu^{-} \rightarrow e^{-} e^{+} e^{-}\right)=m_{\mu}^{5} \frac{\left(g_{e e} g_{\mu e}\right)^{2}}{384 \pi^{3} M_{Z^{\prime}}^{4}}
$$


This process sets therefore bounds on the product of diagonal $\left(g_{e e}\right)$ and non diagonal $\left(g_{\mu e}\right)$ couplings. In the following we assume that $g_{e e}$, is sufficiently small, i.e. it respects the bounds coming from $B . R<10^{-12}$ and we focus our attention on the non diagonal couplings. For instance if $g_{\mu e} \approx 10^{-2}$ (see figure 2), we obtain $g_{e e}<10^{-3}$.

\section{$4 \quad \mathrm{e}^{+} \boldsymbol{\mu}^{-} \rightarrow \mathrm{e}^{-} \boldsymbol{\mu}^{+}$and $\mathrm{e}^{-} \boldsymbol{\mu}^{+} \rightarrow \mathrm{e}^{+} \boldsymbol{\mu}^{+}$}

The $\mathrm{e}^{+(-)} \mu^{-(+)} \rightarrow \mathrm{e}^{-(+)} \mu^{+(-)}$transition violates lepton flavor by two units and is sensitive only to $\mathrm{g}_{\mu e}^{e R}$ and $\mathrm{g}_{\mu e}^{e L}$. The cross section can be computed as the sum of three different contributions corresponding to the exchange of a $Z$ ' in the s channel, in the t channel and to the interference between the two, see figure 3. In general, the cross sections for the LFVviolating processes we consider in the present paper are obtained by summing Z' exchange in s- and t- channel (see figure 3). Considering the process $12 \rightarrow 34$ and indicating with $g_{i j}^{R}, g_{i j}^{L}$ the relevant Z'-fermion-antifermion couplings, the differential cross section in the scattering angle $\theta=\hat{p}_{1} \cdot \hat{p}_{3}$ is the sum of three contributions coming from s-channel square, t-channel squared and s-t interference:

$$
\begin{aligned}
& s \frac{d \sigma_{s s}}{d \cos \theta}=\frac{s^{2}}{8 \pi\left(s-M^{2}\right)^{2}}\left\{\frac{t^{2}}{s^{2}}\left[\left(g_{12}^{R}\right)^{2}\left(g_{34}^{L}\right)^{2}+\left(g_{12}^{L}\right)^{2}\left(g_{34}^{R}\right)^{2}\right]+\frac{u^{2}}{s^{2}}\left[\left(g_{12}^{L}\right)^{2}\left(g_{34}^{L}\right)^{2}+\left(g_{12}^{R}\right)^{2}\left(g_{34}^{R}\right)^{2}\right]\right\} \\
& s \frac{d \sigma_{t t}}{d \cos \theta}=\frac{s^{2}}{8 \pi\left(t-M^{2}\right)^{2}}\left\{\left[\left(g_{13}^{R}\right)^{2}\left(g_{24}^{L}\right)^{2}+\left(g_{13}^{L}\right)^{2}\left(g_{24}^{R}\right)^{2}\right]+\frac{u^{2}}{s^{2}}\left[\left(g_{13}^{L}\right)^{2}\left(g_{24}^{L}\right)^{2}+\left(g_{13}^{R}\right)^{2}\left(g_{24}^{R}\right)^{2}\right]\right\} \\
& s \frac{d \sigma_{s t}}{d \cos \theta}=\frac{s^{2}}{4 \pi\left(s-M^{2}\right)\left(M^{2}-t\right)}\left\{\frac{u^{2}}{s^{2}}\left[g_{12}^{L} g_{13}^{L} g_{24}^{L} g_{34}^{L}+g_{12}^{R} g_{13}^{R} g_{24}^{R} g_{34}^{R}\right]\right\}
\end{aligned}
$$

The expression for the total cross section is rather cumbersome, but it simplifies in the case $g_{i j}^{R}=g_{i j}^{L}=g \forall i, j$ :

$$
\sigma_{\mathrm{TOT}}=\frac{g^{4}}{3 \pi M^{2}} \frac{6 \rho^{2}(1-\rho)(1+\rho)^{2} \log \frac{1+\rho}{\rho}+6 \rho^{4}+3 \rho^{3}-5 \rho^{2}-5 \rho+3}{(1-\rho)^{2}(1+\rho)} ; \quad \rho \equiv \frac{M^{2}}{s}
$$

In the case of heavy $Z^{\prime}, \sqrt{s} \ll M_{Z^{\prime}}$, this expression further simplifies to the following approximate one:

$$
\sigma_{\mathrm{TOT}}=\frac{2 g^{4}}{3 \pi} \frac{s}{M_{Z^{\prime}}^{4}}+\mathcal{O}\left(\frac{1}{M_{Z^{\prime}}^{6}}\right)
$$

The cross section for the process $\mathrm{e}^{+(-)} \mu^{-(+)} \rightarrow \mathrm{e}^{-(+)} \mu^{+(-)}$can be computed using (4.1)-(4.3) with $1=e^{-}, 2=\mu^{+}, 3=\mu^{-}, 4=e^{+}$. If we further assume left and right couplings to be equal $\left(g_{\mu e}^{L}=g_{\mu e}^{R} \equiv g\right)$ we can compute the single event sensitivity (s.e.s.) of our proposed experiment, defined as the value of $g$ such to have 1 event/year. As an example we plot in figure 2 the s.e.s. as a function of the Z' mass at our $\mu-e$ collider, assuming two possible c.m. energy $\sqrt{s}=500 \mathrm{GeV}$ and $1 \mathrm{TeV}$, and luminosity $\mathcal{L}=10^{34} \mathrm{~cm}^{-2} \mathrm{~s}^{-1}$. In both cases, the explorable region of couplings lies a factor $2-3$ below 


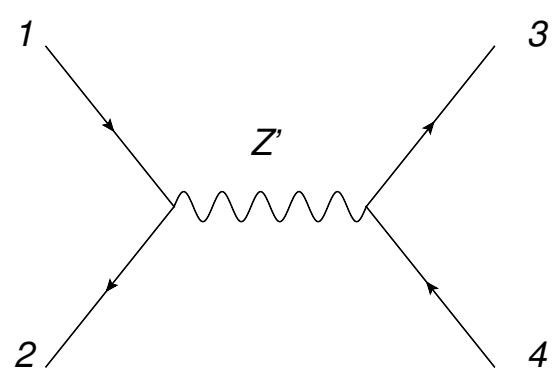

(a)

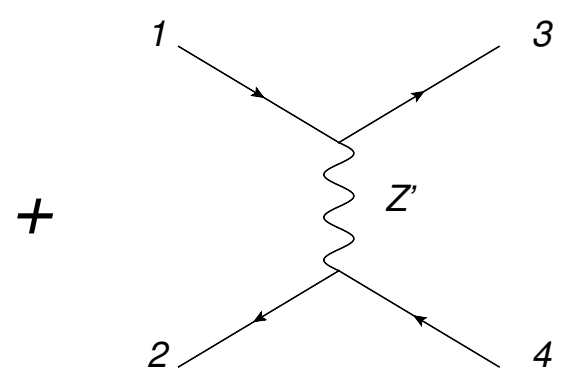

(b)

Figure 3. s- and t-channels contributions to the amplitude for a generic scattering cross sections.

the pessimistic limit set by the standard muon decay. ${ }^{2}$ Here, we do not make any assumption on the actual detection efficiency; we note only, however, that it should be taken not below $\sim 50 \%$, with this requirement being less stringent with increasing c.m. energies.

The observables discussed until now are not sensitive directly to the Z' mass, but rather to the combination $\frac{g}{M_{Z^{\prime}}}$ (see (3.1), (3.4)). We point out that the angular distribution of the outgoing (anti)muon is, instead, sensitive to the value of the Z' mass alone. This can be seen from figure 4 where this distribution in the laboratory frame of a colliding muon of energy $3 \mathrm{TeV}$ and an electron of energy $200 \mathrm{GeV}$ is plotted. In order to emphasize the dependence on the Z' mass we take the values of $M_{Z^{\prime}}=3 \mathrm{TeV}$ for the blue curve and $M_{Z^{\prime}}=100 \mathrm{GeV}$ for the red curve. The reason for the pronounced dependence on the Z' mass is the following. The amplitude is the sum of s-channel contribution, proportional to $1 /\left(M_{Z^{\prime}}^{2}-s\right)$, and t-channel proportional to $1 /\left(M_{Z^{\prime}}^{2}-t\right)$, with $-s \leq t \leq 0$. If the $\mathrm{Z}^{\prime}$ is heavy, $M_{Z^{\prime}}^{2} \gg s$, both amplitudes are proportional to $1 / M_{Z^{\prime}}^{2}$ and angle-independent, therefore the angular distribution is basically flat (blue curve). Indeed, from (4.1), (4.2), (4.3) for couplings all equal to $g$, we obtain:

$$
\frac{d \sigma_{\mathrm{tot}}}{d \cos \theta}=\frac{g^{4} s}{4 \pi M_{Z^{\prime}}^{4}}\left(1+\frac{(1-\cos \theta)^{2}}{4}\right)
$$

which is a rather smooth angular distribution. If instead the $\mathrm{Z}^{\prime}$ is light, $M_{Z^{\prime}}^{2} \ll s$, then for small angles the distribution is dominated by the t-channel amplitude, which is very peaked in the forward direction being roughly proportional to $1 / t$ where $t=-s / 2(1-\cos \theta)$ goes to 0 with the scattering angle. In this case we obtain:

$$
\frac{d \sigma_{\mathrm{tot}}}{d \cos \theta} \approx \frac{g^{4} s}{4 \pi M_{Z^{\prime}}^{4}} \frac{1}{\left(\frac{1}{2}(1-\cos \theta)+\frac{M_{Z^{\prime}}^{2}}{s}\right)^{2}}
$$

which is instead, for small values of $\frac{M_{Z^{\prime}}^{2}}{s}$, strongly peaked at $\theta \approx 0$.

\footnotetext{
${ }^{2}$ We call the limit given by the red dashed line in figure 2 'pessimistic' since a more detailed analysys including the possibility of different left and right couplings, a refined estimate of the calculation of the impact on precision electroweak tests would push the red line towards higher values.
} 


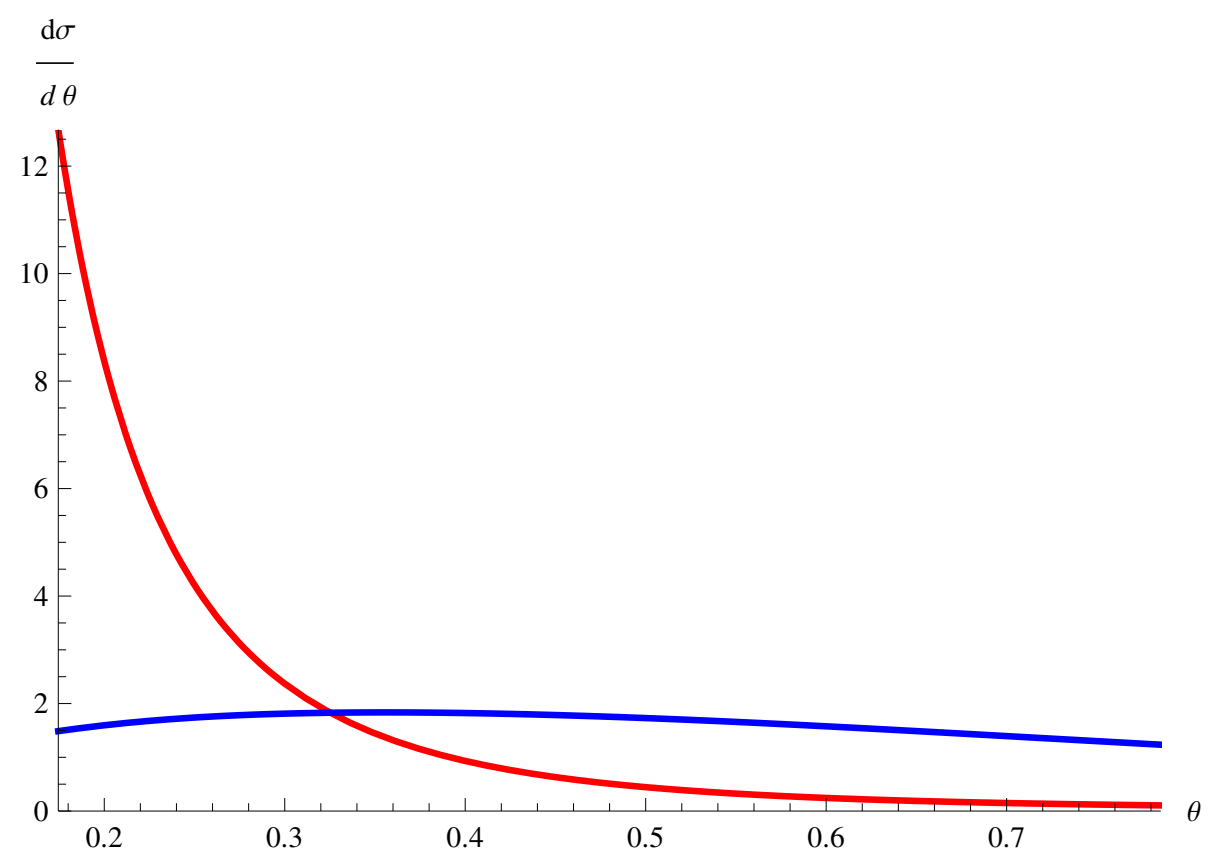

Figure 4. Muon angular distribution for $\mathrm{e}^{+(-)} \mu^{-(+)} \rightarrow \mathrm{e}^{-(+)} \mu^{+(-)}$in the lab frame, where the minitial muon energy is $3 \mathrm{TeVs}$ and the initial electron one is $200 \mathrm{GeVs}$. The red curve corresponds to a Z' mass of $100 \mathrm{GeV}$, while the blue one corresponds to a Z' mass of $3 \mathrm{TeVs}$.

\section{Other processes}

We want now to briefly comment on the possibility to perform two other experiments.

Firstly, we consider the possibility to observe the LFV process $\mathrm{e}^{+(-)} \mu^{-(+)} \rightarrow \mathrm{e}^{+} e^{-}$at the proposed collider. This transition is crossing-connected to the decay $\mu^{-} \rightarrow e^{-} e^{-} e^{+}$, that therefore imposes strong bounds on the couplings. Let us assume $g_{\mu e}^{L, R}=g_{\mu e}$ and $g_{e e}^{L, R}=g_{e e}$; moreover $\sqrt{s} \ll M_{Z^{\prime}}$. Using equation (3.4) and imposing the experimental limit on the $\mu^{-} \rightarrow e^{-} e^{-} e^{+}$branching ratio $(\overline{B R})$, we get:

$$
\frac{g_{e e}^{2} g_{\mu e}^{2}}{2 G_{F}^{2} M_{Z^{\prime}}^{4}}<\overline{B R}, \quad \sigma\left(e^{+(-)} \mu^{-(+)} \rightarrow e^{+} e^{-}\right)=\frac{2 g_{e e}^{2} g_{\mu e}^{2} s}{3 \pi M_{Z^{\prime}}^{4}} \Rightarrow \sigma<\frac{4}{3 \pi} \overline{B R} G_{F}^{2} s
$$

We see that, for instance, for $\sqrt{s}=500 \mathrm{GeV}, \sigma \lesssim 0.5 \times 10^{-8} \mathrm{pb}$, which makes the process impossible to be observed for any reasonable value of the collider's luminosity. Notice that this holds true whatever the value of the couplings: indeed, both the cross sections and the branching ratios depend on the same combination of couplings.

Secondly, we discuss the potential for observing the process $\mathrm{e}^{+(-)} \mu^{-(+)} \rightarrow \mathrm{e}^{-(+)} \mu^{+(-)}$ with a fixed target experiment using presently available muon beams. We take as a reference the setup of [25]. Here, $150 \mathrm{GeV}$ muons impinge on the atomic electrons of a Be target, with total thickness of $60 \mathrm{~cm}$. The beam flux is $\sim 5 \times 10^{7} \mu / \mathrm{s}$. With respect to [25], we further assume that a magnetic spectrometer allows the discrimination between particles of different charge. Given the numbers quoted above the c.m.e of the process is $400 \mathrm{MeV}$ and the experiment's luminosity $\sim 10^{33} \mathrm{~cm}^{-2} \mathrm{~s}^{-1}$. We must here impose the bounds coming 
from standard muon decay, similarly to what has been done in the previous section for the high c.m. energy case. Unfortunately in this case the bounds thus obtained for the values of the couplings are at least one order of magnitude smaller than those required to have one event per year. ${ }^{3}$ Therefore (maybe unless the luminosity is strongly improved), the LFV processes we consider here are unobservable at present day colliders.

\section{Conclusions}

In recent years, relevant progresses have been made in the design for a future muon collider capable of reaching center of mass energies of several TeV. This machine, which would allow the search of phenomena beyond the Standard Model into a so far unexplored energy domain, requires producing very intense, high energy muon beams, with collimation characteristics never obtained to date. We suggest that these beams can also be used to produce electron-muon collisions at very high energies, with luminosity exceeding $10^{34}$ $\mathrm{cm}^{-2} \mathrm{~s}^{-1}$. We have shown that such a machine, would allow studying the lepton flavor violating process $\mathrm{e}^{+-} \mu^{-+} \rightarrow \mathrm{e}^{-+} \mu^{+-}$with a sensitivity potentially better than the one reached by present or proposed rare decay experiments. We have also studied the possibility of performing the same experiment exploiting presently available muon beams, such as that used by the MuonE experiment; we have shown that, unfortunately, in this case the reachable luminosity is at least one order of magnitude smaller than that required to unequivocally observe the signal of our interest. Finally, the abovementioned process, that violates LF by 2 units, looks like the most promising one. For instance, processes that violate LF by 1 unit like $e^{-} \mu^{+} \rightarrow e^{-} e^{+}$, are impossible to observe given the bounds coming from present day experiments.

Open Access. This article is distributed under the terms of the Creative Commons Attribution License (CC-BY 4.0), which permits any use, distribution and reproduction in any medium, provided the original author(s) and source are credited.

\section{References}

[1] N. Doble, L. Gatignon,G. von Holtey and F. Novokoltsev, The upgraded muon beam at the SPS, Nucl. Instrum. Meth. A 311 (1992) 351.

[2] D. Denisov, V. Evdokimov, S. Lukić and P. Ujić, Muon Beam at the Fermilab Test Beam Area, arXiv: 1608.03453 [INSPIRE].

[3] Y. Miyake et al., J-PARC muon source, MUSE, Nucl. Instrum. Meth. A 600 (2009) 22 [INSPIRE].

[4] M. Boscolo, J.-P. Delahaye and M. Palmer, The future prospects of muon colliders and neutrino factories, in Reviews of Accelerator Science and Technology: Volume 10: The Future of Accelerators, pp. 189-214 (2019), DOI [arXiv: 1808.01858] [INSPIRE].

\footnotetext{
${ }^{3} \mathrm{~A}$ new experiment has also been proposed in [26] where the process $\mu^{-} e^{-} \rightarrow e^{-} e^{-}$is enhanced by $(Z-1)^{3}, Z$ being the atomic number. However no additional experimental result can change the conclusions of this section.
} 
[5] M.A. Palmer, Muon Accelerator Program (MAP), http://map.fnal.gov.

[6] M.-H. Wang, Y. Nosochkov, Y. Cai and M. Palmer, Design of a 6 TeV Muon Collider, 2016 JINST 11 P09003 [INSPIRE].

[7] M. Antonelli, M. Boscolo, R. Di Nardo and P. Raimondi, Novel proposal for a low emittance muon beam using positron beam on target, Nucl. Instrum. Meth. A 807 (2016) 101 [arXiv: 1509.04454] [INSPIRE].

[8] MICE collaboration, Demonstration of cooling by the Muon Ionization Cooling Experiment, Nature 578 (2020) 53 [arXiv: 1907.08562] [INSPIRE].

[9] D. Brandt, H. Burkhardt, M. Lamont, S. Myers and J. Wenninger, Accelerator physics at LEP, Rept. Prog. Phys. 63 (2000) 939 [inSPIRE].

[10] ILC collaboration, The International Linear Collider Technical Design Report - Volume 3.II: Accelerator Baseline Design, arXiv:1306.6328 [INSPIRE].

[11] FCC collaboration, FCC-ee: The Lepton Collider: Future Circular Collider Conceptual Design Report Volume 2, Eur. Phys. J. ST 228 (2019) 261 [InSPIRE].

[12] CePC Study Group collaboration, CEPC Conceptual Design Report: Volume 1 Accelerator, arXiv:1809.00285 [INSPIRE].

[13] W. Altmannshofer, C.-Y. Chen, P.S. Bhupal Dev and A. Soni, Lepton flavor violating Z' explanation of the muon anomalous magnetic moment, Phys. Lett. B 762 (2016) 389 [arXiv: 1607.06832] [INSPIRE].

[14] A. De Gouvêa, W.-C. Huang, J. König and M. Sen, Accessible Lepton-Number-Violating Models and Negligible Neutrino Masses, Phys. Rev. D 100 (2019) 075033 [arXiv: 1907.02541] [INSPIRE].

[15] P.S.B. Dev, W. Rodejohann, X.-J. Xu and Y. Zhang, MUonE sensitivity to new physics explanations of the muon anomalous magnetic moment, JHEP 05 (2020) 053 [arXiv: 2002.04822] [INSPIRE].

[16] M. Lindner, M. Platscher and F.S. Queiroz, A Call for New Physics: The Muon Anomalous Magnetic Moment and Lepton Flavor Violation, Phys. Rept. 731 (2018) 1 [arXiv: 1610.06587] [INSPIRE].

[17] L. Calibbi and G. Signorelli, Charged Lepton Flavour Violation: An Experimental and Theoretical Introduction, Riv. Nuovo Cim. 41 (2018) 71 [arXiv:1709.00294] [InSPIRE].

[18] LHCb collaboration, Search for lepton-universality violation in $B^{+} \rightarrow K^{+} \ell^{+} \ell^{-}$decays, Phys. Rev. Lett. 122 (2019) 191801 [arXiv:1903.09252] [INSPIRE].

[19] A. Crivellin, L. Hofer, J. Matias, U. Nierste, S. Pokorski and J. Rosiek, Lepton-flavour violating $B$ decays in generic $Z^{\prime}$ models, Phys. Rev. D 92 (2015) 054013 [arXiv: 1504.07928] [INSPIRE].

[20] G. Feinberg and S. Weinberg, Law of Conservation of Muons, Phys. Rev. Lett. 6 (1961) 381 [INSPIRE].

[21] G. Feinberg and S. Weinberg, Conversion of Muonium into Antimuonium, Phys. Rev. 123 (1961) 1439 [INSPIRE].

[22] A. Masiero, P. Paradisi and M. Passera, New physics at the MUonE experiment at CERN, arXiv: 2002.05418 [INSPIRE]. 
[23] L. Willmann et al., New bounds from searching for muonium to anti-muonium conversion, Phys. Rev. Lett. 82 (1999) 49 [hep-ex/9807011] [InSPIRE].

[24] Particle Data Group collaboration, Review of Particle Physics, Phys. Rev. D 98 (2018) 030001 [INSPIRE].

[25] G. Abbiendi, Letter of Intent: the MUonE project, Tech. Rep. CERN-SPSC-2019-026. SPSC-I-252, CERN, Geneva (Jun, 2019).

[26] M. Koike, Y. Kuno, J. Sato and M. Yamanaka, A new idea to search for charged lepton flavor violation using a muonic atom, Phys. Rev. Lett. 105 (2010) 121601 [arXiv: 1003.1578] [INSPIRE]. 